\title{
Correction to: RNF213 p.Arg4810Lys Heterozygosity in Moyamoya Disease Indicates Early Onset and Bilateral Cerebrovascular Events
}

\author{
Daiichiro Ishigami ${ }^{1} \cdot$ Satoru Miyawaki ${ }^{1} \cdot$ Hideaki Imai $^{2} \cdot$ Masahiro Shimizu $^{3} \cdot$ Hiroki Hongo $^{1} \cdot$ Shogo Dofuku $^{1}$. \\ Kenta Ohara ${ }^{1} \cdot$ Yu Teranishi ${ }^{1}$. Daisuke Shimada ${ }^{4}$. Satoshi Koizumi ${ }^{1} \cdot$ Hideaki Ono $^{5}$. Yudai Hirano ${ }^{1}$. \\ Masafumi Segawa ${ }^{1} \cdot$ Hirofumi Nakatomi ${ }^{1,4} \cdot$ Nobuhito Saito $^{1}$
}

Published online: 26 November 2021

๑) Springer Science+Business Media, LLC, part of Springer Nature 2021

\section{Correction to: Translational Stroke Research https://doi.org/10.1007/s12975-021-00956-8}

In the version of the article originally published, in the sentence beginning "In addition, as described below, a key difference exists...", the superscript number following "Miyatake et al." was unnecessary. In addition, the last sentence in Ethics Approval and Consent to Participate ("Declarations" section) should have read, "Written informed consent was obtained from all the participants or parents/legal guardians for participants under the age of 18."

The original article has been corrected.

Publisher's Note Springer Nature remains neutral with regard to jurisdictional claims in published maps and institutional affiliations.

The original article can be found online at https://doi.org/10.1007/ s12975-021-00956-8.

Satoru Miyawaki

smiya-nsu@m.u-tokyo.ac.jp

1 Department of Neurosurgery, Faculty of Medicine, The University of Tokyo, Tokyo, Japan

2 Department of Neurosurgery, JCHO Tokyo Shinjuku Medical Center, Tokyo, Japan

3 Department of Neurosurgery, Kanto Neurosurgery Hospital, Saitama, Japan

4 Department of Neurosurgery, Kyorin University, Tokyo, Japan

5 Department of Neurosurgery, Fuji Brain Institute and Hospital, Shizuoka, Japan 